\title{
Pelvic organ prolapse and urogynecological assessment in women with spinal cord injury
}

\author{
Marlene Elmelund $\mathbb{D}^{1,2} \cdot$ Fin Biering-Sørensen ${ }^{1} \cdot$ Mette Hornum Bing ${ }^{2} \cdot$ Niels Klarskov $^{2}$
}

Received: 18 May 2018 / Revised: 2 July 2018 / Accepted: 2 July 2018 / Published online: 10 August 2018

(c) International Spinal Cord Society 2018

\begin{abstract}
Study design Observational cross-sectional study.

Objectives Due to weakened pelvic floor muscles, the risk of pelvic organ prolapse (POP) may increase after a spinal cord injury (SCI); hence, the aim of this study was to investigate the occurrence of POP after SCI and to evaluate the need for urogynecological consultations offered to women with SCI.

Setting Clinic for Spinal Cord Injuries, Rigshospitalet, Denmark.

Methods Women with SCI who visited our SCI-clinic during January 2013-January 2018 were offered a specialized urogynecological consultation. Any symptoms of POP, urinary/fecal incontinence, or bladder/bowel emptying problems were registered, and POP was classified according to the POP quantification system during a pelvic examination. Differences in baseline characteristics between women with POP stage $0-1$ and POP stage $\geq 2$ were investigated.

Results A total of 98 women were included in the study. Fourteen women (14\%) reported POP symptoms and 21 women $(21 \%)$ had anatomical POP stage $\geq 2$. The group with POP stage $\geq 2$ had a significantly higher age, higher parity, more with vaginal delivery, and more postmenopausal women, but the groups did not differ on median time after injury, neurological level, and completeness of injury. A total of $71 \%$ experienced urinary incontinence, $27 \%$ experienced fecal incontinence, $63 \%$ experienced bladder emptying problems, and 70\% experienced bowel emptying problems. Consequently, $65 \%$ received treatment.

Conclusions Women with SCI are not in increased risk of developing anatomical POP. Nonetheless, the high occurrence of other urogynecological issues and the high treatment-rate supports the need for specialized urogynecological consultations offered to women with SCI.
\end{abstract}

\section{Introduction}

It is well-known that women with mobility impairments are less likely to receive adequate gynecological screening and care [1]. This, combined with a changed sensibility after injury, raises a concern if women with spinal cord injury (SCI) are in higher risk of having undiagnosed gynecological disorders than able-bodied women.

Marlene Elmelund

marleneelmelund@hotmail.com

1 Clinic for Spinal Cord Injuries, Rigshospitalet, University of Copenhagen, Copenhagen, Denmark

2 Department of Obstetrics and Gynecology, Herlev and Gentofte Hospital, University of Copenhagen, Herlev, Denmark
Pelvic organ prolapse (POP) is a common anatomical condition, affecting up to $50 \%$ of an adult female population, and it is well-documented that the risk of POP increases with advancing age, parity, and vaginal births [2]. In a case report by Wan and Liu [3], the story of a 37-year old nulliparous spinal cord injured woman with a stage 3 uterine prolapse was brought to attention, and it has been questioned if the risk of POP increases after a SCI due to weakness of the pelvic floor muscles, peripheral nerve damage and potential use of increased abdominal pressure during bladder and bowel emptying [3, 4]. Though POP is a benign condition in able-bodied women, the complications of POP in women with SCI may be more comprehensive as the SCI-associated bladder emptying problems and potential urinary retention may be aggravated by an obstructing POP, that can go unnoticed due to reduces sensibility. Nonetheless, there is a complete knowledge-gap regarding POP in women with SCI [5]. 
The Clinic for Spinal Cord Injuries, Rigshospitalet is one of two clinics in Denmark where all persons with SCI are followed every second year from the time of diagnosis. Besides regular consultations with SCI-specialized physicians, the follow-up program includes standardized urological evaluation and treatment of lower urinary tract complications. However, urogynecological assessments in the women with SCI is not a part of the follow-up program. Hence, the aim of this study was to evaluate if specialized urogynecological consultations and treatments should be offered to women with SCI in addition to the standardized urological and SCI-specialized follow-up program. A secondary aim was to investigate the occurrence of POP and other urogynecological issues in women with SCI and to examine risk factors associated with anatomical POP.

\section{Methods}

\section{Study design and participants}

While attending a routine follow-up consultation or during the primary admission after the injury in the Clinic for Spinal Cord Injuries, Rigshospitalet between January 2013 and January 2018, women with SCI were offered a gynecological consultation and examination by their treating physician. The gynecological consultation and examination would take place at a specialized urogynecological department, where the woman was asked if she wanted to participate in the study. This observational cross-sectional study was approved by the Danish Health Authorities and all participant provided written informed consent. Some of the women participating in this study were also included in a recently published study, investigating the prevalence UI in women with SCI from the Clinic for Spinal Cord Injuries, Rigshospitalet by use of information from the clinic's database [6].

\section{The consultation and examination}

The consultation was conducted primarily by a senior doctor specialized in the field of urogynecology. A nurse was present during the examination, and equipment facilitating transfer to and from the examination table was available. During the consultation, a medical history was obtained including parity, mode of delivery, menstrual status, previous urogynecological surgery, and current medication. In addition, the woman was asked if she ever experienced a bulge, lump, or something coming down or falling out through the vagina. If she answered yes, she was registered with symptoms of POP [7]. In addition, she was asked if she ever experienced any involuntary loss of urine (registered as symptoms of UI), any sudden compelling desire to pass urine which is difficult to defer (registered as symptoms of urgency) or any involuntary loss of feces (registered as fecal incontinence) [7]. Prior to the consultation, the woman was also asked to fill out a Danish translation of the validated International Consultation on Incontinence questionnaire, urinary incontinence, short form (ICIQ-UI-SF) [8], and a questionnaire regarding the severity of POP symptoms, originating from the internationally validated International Consultation on Incontinence questionnaire, vaginal symptoms (ICIQ-VS) [9]. Both questionnaires contain three sub-questions regarding the frequency, severity and impact of UI, or POP on quality of life, resulting in a total score ranging from 0-21 for the UI-questionnaire and $0-17$ for the POP questionnaire with a higher score indicating more severe symptoms. In addition, the situations in which UI occurred were registered in the ICIQ-UI-SF questionnaire. If the woman reported of UI during effort or physical activity or on sneezing or coughing, she was diagnosed with symptomatic stress UI. Further, bladder emptying problems were registered if the woman answered yes when asked if she experienced any difficulties with voiding and/or if she used a primary bladder emptying method other than normal according to the International SCI lower urinary tract function basic data set [10]. Bowel emptying problems were registered if the woman answered yes when asked if she experienced any difficulties with bowel emptying. Finally, the woman was asked if she had a partner and if she was currently sexually active.

A pelvic examination was conducted with the woman in a supine position and included inspection of the vulva, vagina, and cervix, bimanual palpation, and an intravaginal ultrasound examination of the internal pelvic organs. The pelvic floor muscle strength was evaluated by vaginal palpation during voluntary pelvic floor muscle contraction. The muscle strength was classified as strong, normal, weak, or absent [7]. The anatomical definition of the sign of POP was evaluated during abdominal straining, and POP was classified in each vaginal compartment according to the simplified technique of the POP quantification system as described by Swift et al. [11]. The compartment with the largest stage of POP was reported in the results.

Information was obtained from medical records regarding the date, etiology, neurological level, and completeness of injury according to the American Spinal Injury Association Impairment Scale (AIS) [12-14]. Information from the most recent cytological cervical cancer screenings test was obtained. If the woman could not recall the date, this was obtained from a national pathological database. If a cervical cytology sample had not been collected within the last three years for women aged 23-49 years or within the last five years for women aged 50-64 years, as recommended in the Danish cercival cancer screenings program [15], the sample was collected during the examination. 
When indicated, additional diagnostic tests were conducted and relevant treatments were offered.

\section{Statistical analyses}

The distribution of patient-reported urogynecological issues according to completeness of injury was tested using Fisher's exact test, and differences in characteristics between women with POP stage $0-1$ and POP stage $\geq 2$ were investigated using Student's $t$-test, Mann-Whitney $U$ test or Fisher's exact test. A bivariate logistic regression analysis was conducted in a subgroup of women with a history of vaginal delivery. The outcome was risk of POP stage $\geq 2$, and the included parameters were age and followup period divided by $<1$ year, $1-5$ years, and $>5$ years after injury. The assumption of linearity was tested for the variable age by including a quadratic value of age in the model. All analyses were conducted using SAS version 7.1 (SAS Institute Inc, Cary, NC, USA), and a $p$-value $<0.05$ was considered statistically significant.

\section{Results}

\section{Study population}

Between January 2013 and January 2018, 99 women with SCI attended the urogynecological consultation. One woman declined to participate during the consultation, and 98 women were included in the study. Demographics on the included women are shown in Table 1. During this period, $\sim 700$ women with SCI were listed as patients in the Clinic. The women included in this study were comparable with the complete group of women in terms of classification of injury (catagorized by neurological level and completeness of injury) and median age at injury (44 years vs 47 years in this study).

Senior medical doctors specialized in the field of urogynecology consulted 96 of the 98 women of whom 91 women were consulted by the same two senior doctors. Two women attended a consultation with a medical doctor in gynecological training. Among the included women, 41 had not been gynecologically examined after the onset of injury. The follow-up period ranged between 2 months and 61 years after SCI.

\section{Patient-reported symptoms}

Patient-reported urogynecological symptoms are shown in Fig. 1. The most frequently reported symptoms were bowel emptying problems $(70 \%)$, bladder emptying problems (63\%), UI (71\%), and urgency (49\%). Of the 70 urinary incontinent women, 44 presented with symptoms of stress
Table 1 Demographic information on the 98 included women

\begin{tabular}{|c|c|}
\hline Median age (years) & $54(40-67)$ \\
\hline Median age at injury (years) & $47(24-64)$ \\
\hline Median follow-up after injury (years) & $\begin{array}{l}2.3 \\
(0.6-9.6)\end{array}$ \\
\hline Mean body mass index $\left(\mathrm{kg} / \mathrm{m}^{2}\right)$ & $26.3( \pm 5.9)$ \\
\hline Median parity & $2(0-2)$ \\
\hline Nullipara & $25(26 \%)$ \\
\hline History of vaginal delivery & $60(61 \%)$ \\
\hline Women with parity after injury ${ }^{a}$ & $9(9 \%)$ \\
\hline Menopause & $58(59 \%)$ \\
\hline Hysterectomy & $8(8 \%)$ \\
\hline Previous prolapse surgery & $4(4 \%)$ \\
\hline Receiving treatment for $\mathrm{UI}^{\mathrm{b}}$ & $22(22 \%)$ \\
\hline Receiving HRT & $2(2 \%)$ \\
\hline \multicolumn{2}{|l|}{ Neurological level of injury } \\
\hline Cervical & $34(37 \%)$ \\
\hline Thoracic & $29(32 \%)$ \\
\hline Lumbar & $26(29 \%)$ \\
\hline Sacral & $2(2 \%)$ \\
\hline \multicolumn{2}{|l|}{ Completeness (AIS) } \\
\hline A & $9(11 \%)$ \\
\hline B & $1(1 \%)$ \\
\hline $\mathrm{C}$ & $7(8 \%)$ \\
\hline $\mathrm{D}$ & $68(80 \%)$ \\
\hline \multicolumn{2}{|l|}{ Classification of injury by NLI and AIS } \\
\hline C1-C8, AIS ABC & $6(7 \%)$ \\
\hline Th1-S5, AIS ABC & $11(13 \%)$ \\
\hline Any NLI, AIS D & $68(80 \%)$ \\
\hline \multicolumn{2}{|l|}{ Etiology of injury } \\
\hline Traumatic, sports & $2(2 \%)$ \\
\hline Traumatic, assault & 0 \\
\hline Traumatic, transport & $9(9 \%)$ \\
\hline Traumatic, fall & $11(11 \%)$ \\
\hline Traumatic, other cause & $7(7 \%)$ \\
\hline Non-traumatic, congenital & $11(11 \%)$ \\
\hline Non-traumatic, degenerative & $14(14 \%)$ \\
\hline Non-traumatic, benign tumor & $13(13 \%)$ \\
\hline Non-traumatic, malignant tumor & $3(3 \%)$ \\
\hline Non-traumatic, vascular & $11(11 \%)$ \\
\hline Non-traumatic, infection & $7(7 \%)$ \\
\hline Non-traumatic, other cause & $10(10 \%)$ \\
\hline \multicolumn{2}{|l|}{ Mobility } \\
\hline Walks without walking aids & $26(27 \%)$ \\
\hline Walks with walking aids & $55(56 \%)$ \\
\hline Permanent wheelchair user & $17(17 \%)$ \\
\hline
\end{tabular}

Results are presented in mean $( \pm \mathrm{sd})$, median $(\mathrm{q} 25-\mathrm{q} 75)$ or in total numbers $(\%)$. Data is incomplete regarding NLI $(n=7)$, AIS $(n=13)$, classification of injury by AIS and NLI $(n=13)$ and body mass index $(n=19)$

AIS American Spinal Injury Association impairment scale, NLI neurological level of injury, UI urinary incontinence, HRT hormone replacement therapy

${ }^{a}$ Including five vaginal deliveries and eight cesarean sections.

${ }^{\mathrm{b}}$ Including bladder relaxant drugs $(n=19)$ or vesical botulinum toxin injections within the last year $(n=5)$

UI. Urgency was significantly more frequent among the women with a less complete injury (AIS D), and bladder 
Fig. 1 Patient-reported urogynecological symptoms

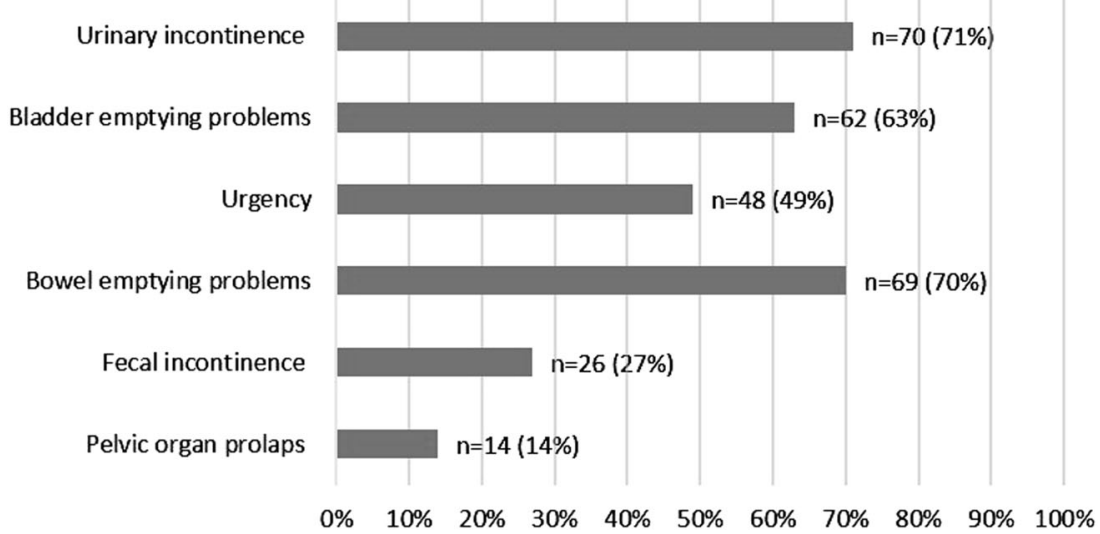

emptying problems were significantly more frequent among the women with a more complete injury (AIS ABC). When the women were asked about the debut of the symptom, $3 \%$ reported of UI prior to the SCI, $4 \%$ had urgency prior to the SCI, $8 \%$ had POP symptoms prior to the SCI and no women had fecal incontinence prior to the SCI. Of the 70 incontinent women, 59 had filled out the ICIQ-UI-SF questionnaire. The mean $( \pm \mathrm{SD})$ total score was $12.1( \pm 4.5)$. A total of 14 women reported of symptomatic POP of whom 12 had filled out the POP questionnaire. The median (interquartile range) total score was $10(8.5-13)$. When asked about their bladder emptying methods as described in the International SCI lower urinary tract function basic data set [10], 39 (40\%) used normal bladder emptying, 31 (32\%) used clean intermittent catheterization, $10(10 \%)$ used abdominal expression, 5 (5\%) used reflex triggering, 8 (8\%) had a suprapubic catheter, and $5(5 \%)$ had a urethral cathether. In this case, normal bladder emptying method refers to voluntary initiation of micturition without reflex stimulation or compression, but does not presume entirely normal bladder function. A total of 54 women $(55 \%)$ had a partner and 38 women (39\%) were sexually active.

\section{Objective findings, diagnostics, and treatments}

Objective gynecological findings, diagnostics, and treatments are presented in Table 2 and Table 3. The pelvic floor muscle strength was evaluated in 90 of the 98 women and was absent in $21 \%$, weak in $41 \%$, normal in $24 \%$, and strong in 13\%. Among the women aged 23-64 years $(n=$ $60), 18(30 \%)$ had not followed the recommended cervical cancer screening program. A total of 48 cervical cytology samples, four biopsies, one colcoscopy, and one ovarian cancer screening with antigen CA-125 were carried out; none led to further diagnostics and treatments.

Overall, 34 women $(35 \%)$ received no treatment or only counseling, 56 women (57\%) received conservative treatment including medication or other conservative treatments as described in Table 3, and eight women (8\%) received a
Table 2 Objective gynecological findings

\begin{tabular}{ll}
\hline Pelvic examination & \\
\hline Pelvic organ prolapse stage $\geq 2$ & $21(21 \%)$ \\
$\quad$ Anterior compartment & $11(11 \%)$ \\
$\quad$ Apical compartment & $3(3 \%)$ \\
$\quad$ Posterior compartment & $15(15 \%)$ \\
Abnormality of external genitals ${ }^{\text {a }}$ & $4(4 \%)$ \\
Vulvar or vaginal ulcers $^{\text {Vaginal atrophic mucosa }}$ & $4(4 \%)$ \\
Other vaginal pathology ${ }^{b}$ & $26(27 \%)$ \\
Cervical pathology ${ }^{c}$ & $6(6 \%)$ \\
Cervical or vaginal polyp & $3(3 \%)$ \\
Rectal prolaps & $4(4 \%)$ \\
No pathology & $1(1 \%)$ \\
\end{tabular}

Ultrasound examination

No pathology

$81(83 \%)$

Ovarian cyst $>30 \mathrm{~mm}$

$1(1 \%)$

Uterin fibroids

$16(16 \%)$

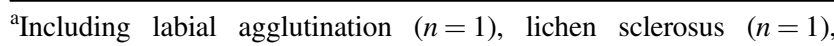
changes after circumsition $(n=1)$, and labia majores hypertrophy $(n=1)$

${ }^{\mathrm{b}}$ Including vaginal erythema $(n=5)$, abnormal vaginal discharge $(n=$ $1)$, and vaginal cyst $(n=1)$

${ }^{\mathrm{c}}$ Including cervical hyperkeratosis $(n=1)$, erythroplakia $(n=1)$, and erosion $(n=1)$

surgical treatment, of whom three women underwent POPrepair surgery (one combined anterior and posterior colporrhaphy and two amputations of the cervix). POP-repair surgery was only conducted in those women who had a strong wish of surgical treatment and where pessary treatment of the POP was insufficient. Two women were bothered by pain and pelvic pressure due to an elongated collum and one woman experienced complications with conducting intermittent catheterization due to the prolapse. All three women expressed satisfaction with the post-operative result. Of the 
Table 3 Diagnostics and treatments

\begin{tabular}{|c|c|}
\hline \multicolumn{2}{|l|}{ Diagnostics } \\
\hline Biopsy ${ }^{a}$ & $4(4 \%)$ \\
\hline Ovarian cancer antigen CA-125 test & $1(1 \%)$ \\
\hline Cervical cytology test & $48(49 \%)$ \\
\hline Colposcopy & $1(1 \%)$ \\
\hline Urine test strip & $8(8 \%)$ \\
\hline Urine or urethral culture & $2(2 \%)$ \\
\hline Residual urine measurement & $12(12 \%)$ \\
\hline Free uroflowmetry & $8(8 \%)$ \\
\hline Cystometry and pressure-flow study & $2(2 \%)$ \\
\hline \multicolumn{2}{|l|}{ Treatments } \\
\hline No treatment ${ }^{b}$ & $34(35 \%)$ \\
\hline Surgery & $8(8 \%)$ \\
\hline Prolapse surgery & $3(3 \%)$ \\
\hline Mid-urethral tape operation & $1(1 \%)$ \\
\hline Urethral injection therapy & $1(1 \%)$ \\
\hline Botulinum toxin injections & $3(3 \%)$ \\
\hline Labia majores reduction $^{\mathrm{c}}$ & $1(1 \%)$ \\
\hline Medication & $40(41 \%)$ \\
\hline Bladder relaxant drugs & $6(6 \%)$ \\
\hline Drugs improving bowel emptying & $2(2 \%)$ \\
\hline Oral antibiotic treatment & $2(2 \%)$ \\
\hline Cutan antibiotics or hormone treatment & $7(7 \%)$ \\
\hline Vaginal estradiol treatment & $31(32 \%)$ \\
\hline Other conservative treatment & $37(38 \%)$ \\
\hline Pessary for vaginal prolapse & $4(4 \%)$ \\
\hline Pessary for urinary incontinence & $12(12 \%)$ \\
\hline Tampons for urinary incontinence & $5(5 \%)$ \\
\hline Pelvic floor muscle training ${ }^{\mathrm{d}}$ & $22(22 \%)$ \\
\hline Intrauterin device insertion/removal & $2(2 \%)$ \\
\hline $\begin{array}{l}\text { Counseling on fluid intake, bladder emptying method, } \\
\text { contraception or pregnancy }\end{array}$ & $17(17 \%)$ \\
\hline
\end{tabular}

${ }^{\mathrm{a} B i o p s y}$ from cervix $(n=2)$, labia majores $(n=1)$, and endometrium $(n=1)$

${ }^{\mathrm{b}}$ Including women who received counseling

${ }^{c}$ Due to labia majora hypertrophy causing bladder emptying problems with clean intermittent catheterization

${ }^{\mathrm{d}}$ Excluding one woman receiving pelvic floor stretching therapy

44 women with symptomatic stress UI, 33 (75\%) received treatment of stress UI including referral to a group or individual pelvic floor muscle training-program with a physiotherapist either at the hospital or locally $(n=15)$, pessary for UI $(n=11)$, tampons for UI $(n=4)$, vaginal estradiol treatment $(n=18)$, and surgery $(n=1)$. The surgical treatment of SUI included intraurethral injection therapy with Bulkamid $^{\circledR}$ in a woman with urodynamically confirmed SUI, acontractile bladder and paresis of the pelvic floor muscles, but the treatment was not effective. As the woman could not carry out intermittent catheterization, she was treated with a tight mid-urethral tape combined with a suprapubic catheter which resulted in complete continence.

\section{Pelvic organ prolapse}

At examination, 21 women (21\%) had POP stage $\geq 2 ; 16$ had POP stage 2 and 5 had POP stage 3. Among the women with a history of vaginal delivery, $70 \%$ had POP stage $0-1$, $22 \%$ had POP stage 2, and $8 \%$ had POP stage 3 compared with $92 \%$ with POP stage $0-1,8 \%$ with POP stage 2 and none with POP stage 3 among the women with no history of vaginal delivery. Of the 21 women with POP stage $\geq 2,12$ experienced symptoms of POP (57\%).

Mean age and parity were significantly higher in women with POP stage $\geq 2$, and more women with POP stage $\geq 2$ were menopausal and had a history of vaginal delivery than women with POP stage $0-1$ (Table 4). In addition, a logistic regression analysis was conducted in a subgroup of women with a history of vaginal delivery $(n=60)$, which included no women with myelomeningocele as etiology of injury. When adjusting to age, the odds of having POP stage $\geq 2$ was 0.47 (95\% CI $0.05-4.77, p=0.5)$ after $1-5$ years follow up and 0.71 (95\% CI 0.16-3.08, $p=0.6)$ after $>5$ years follow up compared with $<1$ year follow up after injury, hence, the risk of having POP stage $\geq 2$ did not increase with increasing time after injury. There was no difference in primary bladder emptying methods between the two groups, where the majority of women used normal bladder emptying or intermittent catheterization in both groups (Fig. 2).

\section{Discussion}

In this observational study, $21 \%$ of women with SCI had anatomical POP stage 2 or more, and POP was associated with the known risk factors age, parity, vaginal delivery, and menopause [2], but not with completeness and level of injury or follow-up period after injury.

Swift investigated the prevalence of anatomical POP according to the POP quantification system in neurologically intact women with a mean age of 44 years and found that $50 \%$ had anatomical POP stage $\geq 2$ [2]. It could be argued that parity and mode of delivery may differ in women with and without a SCI, but even among the women with a history of vaginal delivery, $58 \%$ had POP stage $\geq 2$ in the study by Swift compared with $30 \%$ in the present study. In a group of Brazilian women comparable with this study population on parity, body mass index and mode of delivery but younger of age (mean age 41 years), POP stage $\geq 2$ occurred in $24.5 \%$ [16]. In comparison, anatomical POP is not more prevalent in women with SCI compared with 
Table 4 Characteristics associated with POP stage $\geq 2$

\begin{tabular}{|c|c|c|c|}
\hline Characteristics & $\begin{array}{l}\text { POP stage } 0-1 \\
(n=77)\end{array}$ & $\begin{array}{l}\text { POP stage } \geq 2 \\
(n=21)\end{array}$ & $p$ \\
\hline Mean age (years) & $50.0(45.8-54.1)$ & $61.5(56.4-45.8)$ & 0.0007 \\
\hline Mean age at injury (years) & $40.9(35.6-46.2)$ & $50.3(38.2-62.4)$ & 0.11 \\
\hline $\begin{array}{l}\text { Median follow-up after injury } \\
\text { (years) }\end{array}$ & $2.7(0.8-9.6)$ & $1.0(0.4-5.7)$ & 0.3 \\
\hline Mean body mass index $\left(\mathrm{kg} / \mathrm{m}^{2}\right)$ & $25.9(24.4-27.4)$ & $27.3(24.4-30.3)$ & 0.4 \\
\hline Mean parity & $1.4(1.1-1.6)$ & $2.0(1.5-2.6)$ & 0.014 \\
\hline History of vaginal delivery & & & 0.011 \\
\hline Yes & $42(54 \%)$ & $18(86 \%)$ & \\
\hline No & $35(45 \%)$ & $3(14 \%)$ & \\
\hline Menopause & & & 0.025 \\
\hline Yes & $41(53 \%)$ & $17(81 \%)$ & \\
\hline No & $36(47 \%)$ & $4(19 \%)$ & \\
\hline Hysterectomy & & & 0.20 \\
\hline Yes & $8(10 \%)$ & $0(0 \%)$ & \\
\hline No & $69(90 \%)$ & $21(100 \%)$ & \\
\hline $\begin{array}{l}\text { Classification of injury by NLI } \\
\text { and AIS }\end{array}$ & & & 0.25 \\
\hline C1-C8, AIS ABC & $6(9 \%)$ & $0(0 \%)$ & \\
\hline Th1-S5, AIS ABC & $7(10 \%)$ & $4(24 \%)$ & \\
\hline Any NLI, AIS D & $55(81 \%)$ & $13(76 \%)$ & \\
\hline Etiology of injury & & & 1.0 \\
\hline Spinal cord injury & $68(88 \%)$ & $19(90 \%)$ & \\
\hline Myelomeningocele & $9(12 \%)$ & $2(10 \%)$ & \\
\hline Mobility & & & 0.6 \\
\hline Walks without walking aids & $22(29 \%)$ & $4(19 \%)$ & \\
\hline Walks with walking aids & $41(53 \%)$ & $14(67 \%)$ & \\
\hline Permanent wheelchair user & $14(18 \%)$ & $3(14 \%)$ & \\
\hline $\begin{array}{l}\text { Primary bladder emptying } \\
\text { method }\end{array}$ & & & 0.4 \\
\hline $\begin{array}{l}\text { Bladder expression/ } \\
\text { Valsalva's maneuver }\end{array}$ & $7(9 \%)$ & $3(14 \%)$ & \\
\hline Other method(s) & $70(91 \%)$ & $18(86 \%)$ & \\
\hline Pelvic floor muscle strength & & & 0.20 \\
\hline Absent or weak & $41(59 \%)$ & $15(75 \%)$ & \\
\hline Normal or strong & $29(41 \%)$ & $5(25 \%)$ & \\
\hline Bowel emptying problem & $55(71 \%)$ & $14(67 \%)$ & 0.8 \\
\hline Urinary incontinence & $54(70 \%)$ & $16(76 \%)$ & 0.8 \\
\hline Fecal incontinence & $18(23 \%)$ & $8(38 \%)$ & 0.26 \\
\hline
\end{tabular}

Results are presented in mean (95\% confidence interval), median (q25-q75) or in total numbers (\%)

$P O P$ Pelvic organ prolapse, NLI Neurological level of injury, AIS American Spinal Injury Association impairment scale

neurologically intact women. Additionally, the risk of POP does not increase with increasing time after injury in this study. Even among the women with the longest follow-up period due to a congenital myelomeningocele as etiology of the spinal cord lesion (median follow-up was 40 years, range $18-61$ ), only $18 \%$ had POP, suggesting that a SCI is not a risk indicator of POP.

POP has not previously been investigated in women with SCI, but Dillon et al. investigated the prevalence of POP in 280 women with multiple sclerosis (MS) [17], and found that only $9 \%$ had anatomical POP stage $\geq 2$. Though the evolving symptoms in persons with MS differs fundamentally from the more chronic complications after a SCI, the

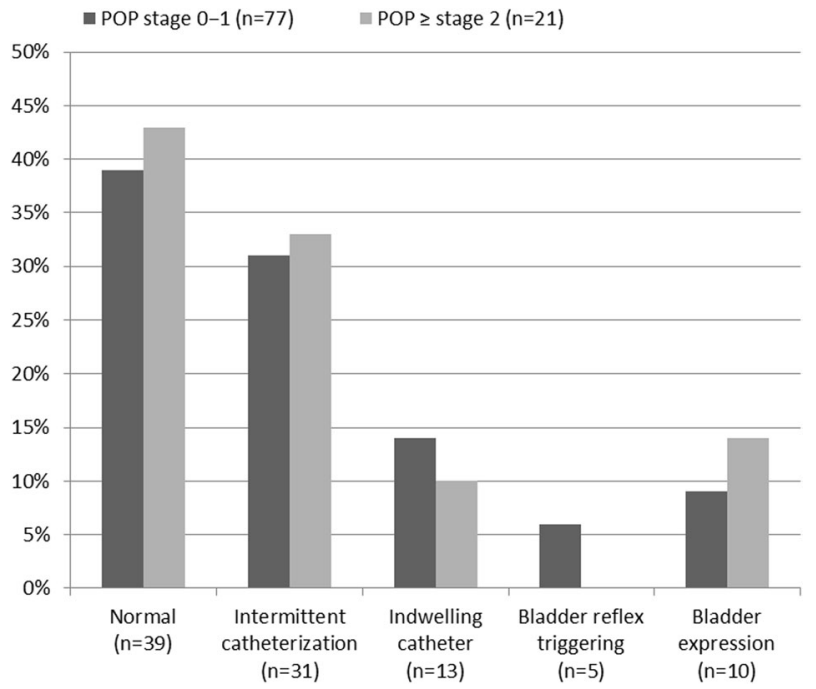

Fig. 2 Anatomical pelvic organ prolapse according to primary bladder emptying method

findings of both studies indicate that women with a neurological disorder have a lower occurrence of POP compared with able-bodied women. We hypothesize that this is due primarily to impaired mobility, where less stress is applied on the pelvic organs, connective tissue, and pelvic floor muscles compared with neurologically intact women. Clinically, these findings are relevant when considering mode of delivery in a pregnant woman with SCI, as the concern of an increased risk of POP after a SCI may be disregarded.

Overall, $70 \%$ of the study population had followed the age-specific cervical cancer screening program. During 2009-2015, the coverage of cervical cancer screening was $75-76 \%$ in Danish women, which is comparable to the coverage found in our study [18]. Previous studies have shown that among women with disabilities, non-ambulatory status and major lower extremity mobility difficulties were associated with an increased risk of non-participation in the cervical cancer screening program [19, 20]. Given that the majority of the spinal cord injured women in this study were ambulatory with a less complete injury, the coverage of the cancer screening program might be lower in completely paralyzed women. No women were diagnosed with dysplasia or gynecological cancer as a consequence of this study, which is not surprising considering the limited size of the study. Nonetheless, physicians should consider women with SCI as a vulnerable population for receipt of cervical cytology tests and regular gynecological health examinations.

The high occurrences of symptomatic UI (71\%), fecal incontinence (27\%), bladder- (63\%), and bowel emptying problems $(70 \%)$ show that the majority of the included women deal with several urogynecological complications 
following the injury. All the included women were followed at the highly specialized Clinic for SCI prior to the consultation, where the bladder function is regularly evaluated using urodynamic investigations. Due to the risk of renal deterioration following a SCI, treatment of neurogenic bladder dysfunction and especially high-pressure detrusor overactivity is highly prioritized in this population [21, 22]. Among the included women, $22 \%$ were already receiving treatment with intravesical botulinum toxin injections or antimuscarinic/beta-3 adrenoceptor agonistic therapy and $45 \%$ used either intermittent catheterization or permanent catheter prior to the consultation. Despite of this, $75 \%$ of the women with symptomatic stress UI received treatment of their incontinence as a consequence of this study, which underlines the value of specialized urogynecological consultations offered to urinary incontinent women with a SCI.

The strength of this study is primarily its novelty as it is the first study to investigate the occurrence of POP in women with SCI. The limitations of the study include the small size, which prevents the use of multivariate analyses, and the relatively short follow-up period. Secondly, there was no age-matched cohort of women undergoing pelvic examination in the study, making the comparisons with able-bodied women somewhat conjectural. Thirdly, the diagnosis of SUI was based on the woman's reported symptoms and not on urodynamic evaluations. With the purpose of identifying detrusor overactivity, urodynamic investigations were already a part of the standardized urological follow-up program in the Clinic for Spinal Cord Injuries, thus, urodynamic evaluations were only conducted in this study when it was clinically relevant, e.g., prior to surgical treatment of UI. Unfortunately, the results from the urodynamic evaluations conducted in the standardized follow-up program were not available in this study. Finally, the number of eligible women who were offered to attend the gynecological consultation but declined or did not show up was not registered. However, 700 women were listed as patients in the Clinic for Spinal Cord Injuries, of whom a seventh was included in this study. It could be questioned if the study population is a representable sample of women with SCI or if the women with urogynecological issues are overrepresented in this study. The same authors conducted a study including the majority of women $(n=609)$ followed at the Clinic for Spinal Cord Injuries [6], and data were collected from a SCI database containing standardized SCIspecific questionnaires, including a validated bladder function questionnaire [10]. In this study, the prevalence of daily to monthly UI within the last three months was $49 \%$, which is lower than the $71 \%$ occurrence of any UI without timelimits found in the present study. However, of the 98 included women in this study 75 were also included in the database study, and in this sample $56 \%$ experienced UI compared with $71 \%$ in the present study. This suggests that the descrepancy between the study-findings is caused primarily by use of different definitions of UI rather than selection bias. Further, the two study populations did not differ on neurological level of injury, completeness of injury, age, and age at injury, but the women in the present study had a shorter median follow-up period after injury (2.3 years) compared with the women in the database study (7.2 years).

In conclusion, the occurrence of anatomical POP in women with SCI is not higher than in neurologically intact women, and the risk of POP does not increase with increasing time after injury. Though anatomical POP does not occur more frequently in women with SCI, other urogynecological issues like UI are highly prevalent, supporting the value of urogynecological consultations and treatments offered to women with SCI. It would be interesting for future studies to investigate the effect of urogynecological interventions on UI and other urogynecological issues, including an evaluation of conservative as well as surgical treatments of in particular stress UI in women with a neurological disorder.

Author contributions ME was responsible for designing and planning the study, data analysis and interpretation, and writing the report. FB-S contributed to the design and planning of the study, interpretation of the results, and provided feedback on the report. MHB was responsible for data acquisition, contributed to interpretation of the results, and provided feedback on the report. NK contributed to the design and planning of the study, data analysis, interpretation of the results, and provided feedback on the report.

\section{Compliance with ethical standards}

Conflict of interest ME and NK have received paid travel expenses from Contura travel expenses from Contura and MB has received paid travel expenses from Normedi. FB-S has no conflicts of interest to disclose.

\section{References}

1. Schopp LH, Sanford TC, Hagglund KJ, Gay JW, Coatney MA. Removing service barriers for women with physical disabilities: promoting accessibility in the gynecologic care setting. J Midwifery Women's Health. 2002;47:74-9.

2. Swift SE. The distribution of pelvic organ support in a population of female subjects seen for routine gynecologic health care. Am J Obstet Gynecol. 2000;183:277-85.

3. Wan L, Liu X. Delayed-onset advanced pelvic organ prolapse after spinal cord injury in a young, nulliparous woman. Int Urogynecol J. 2016;27:825-7.

4. Ruffion A, Chartier-Kastler E. Specific features of genital prolapse in spinal cord injury patients. Prog Urol. 2007;17:440-1.

5. Fletcher S, Nosek MA, Rizk DE. Mobility impairments and pelvic health disorders in women: the need for innovative treatment and research. Int Urogynecol J. 2014;25:1003-4.

6. Elmelund M, Klarskov N, Biering-Sorensen F Prevalence of urinary incontinence in women with spinal cord injury. Spinal 
Cord 2018; e-pub ahead of print 2018; https://doi.org/10.1038/ s41393-018-0157-0

7. Haylen BT, de Ridder D, Freeman RM, Swift SE, Berghmans B, Lee J, et al. An international urogynecological association (iuga)/ international continence society (ics) joint report on the terminology for female pelvic floor dysfunction. Int Urogynecol J. 2010;21:5-26.

8. Avery K, Donovan J, Peters TJ, Shaw C, Gotoh M, Abrams P. Iciq: a brief and robust measure for evaluating the symptoms and impact of urinary incontinence. Neurourol Urodyn. 2004;23:322-30.

9. Price N, Jackson SR, Avery K, Brookes ST, Abrams P. Development and psychometric evaluation of the iciq vaginal symptoms questionnaire: the iciq-vs. BJOG. 2006;113:700-12.

10. Biering-Sorensen F, Craggs M, Kennelly M, Schick E, Wyndaele JJ. International lower urinary tract function basic spinal cord injury data set. Spinal Cord. 2008;46:325-30.

11. Swift S, Morris S, McKinnie V, Freeman R, Petri E, Scotti RJ, et al. Validation of a simplified technique for using the popq pelvic organ prolapse classification system. Int Urogynecol $\mathbf{J}$ Pelvic Floor Dysfunct. 2006;17:615-20.

12. Biering-Sorensen F, DeVivo MJ, Charlifue S, Chen Y, New PW, Noonan V, et al. International spinal cord injury core data set (version 2.0)-including standardization of reporting. Spinal Cord. 2017;55:759-64.

13. DeVivo M, Biering-Sorensen F, Charlifue S, Noonan V, Post M, Stripling $\mathrm{T}$, et al. International spinal cord injury core data set. Spinal Cord. 2006;44:535-40.

14. Kirshblum SC, Burns SP, Biering-Sorensen F, Donovan W, Graves DE, Jha A, et al. International standards for neurological classification of spinal cord injury (revised 2011). J Spinal Cord Med. 2011;34:535-46.

15. Sundhedsstyrrelsen. Screening for cervical cancer - recommendations. Copenhagen: National Board of Health; 2012.

16. Horst W, do Valle JB, Silva JC, Gascho CLL. Pelvic organ prolapse: prevalence and risk factors in a brazilian population. Int Urogynecol J. 2017;28:1165-70.

17. Dillon BE, Seideman CA, Lee D, Greenberg B, Frohman EM, Lemack GE. A surprisingly low prevalence of demonstrable stress urinary incontinence and pelvic organ prolapse in women with multiple sclerosis followed at a tertiary neurogenic bladder clinic. J Urol. 2013;189:976-9.

18. Lynge E, Andersen B, Christensen J, Ejersbo D, Jochumsen K, Johansen $\mathrm{T}$, et al. Cervical screening in denmark-a success followed by stagnation. Acta Oncol. 2018;57:354-61.

19. Cheng E, Myers L, Wolf S, Shatin D, Cui XP, Ellison G, et al. Mobility impairments and use of preventive services in women with multiple sclerosis: Observational study. BMJ. 2001;323:968-9.

20. Iezzoni LI, McCarthy EP, Davis RB, Harris-David L, O’Day B. Use of screening and preventive services among women with disabilities. Am J Med Qual. 2001;16:135-44.

21. Elmelund M, Oturai PS, Toson B, Biering-Sorensen F. Forty-fiveyear follow-up on the renal function after spinal cord injury. Spinal Cord. 2016;54:445-51.

22. Stohrer M, Blok B, Castro-Diaz D, Chartier-Kastler E, Del Popolo $\mathrm{G}$, Kramer G, et al. Eau guidelines on neurogenic lower urinary tract dysfunction. Eur Urol. 2009;56:81-8. 